\title{
ESCUELA DE PSICOLOGÍA EN VENEZUELA: ESTRUCTURA CURRICULAR1
}

\author{
Herminia Vicentelli ${ }^{2}$ \\ Universidad Pedagógica Experimental Libertador Venezuela
}

\begin{abstract}
Resumo
O artigo refere-se à formação acadêmica do psicólogo na Venezuela, estudando a estrutura curricular, tendo como referencial as recomendações, sugestões e considerações emanadas dos eventos que influenciaram o delineamento do ensino da Psicologia e sua atuação profissional.

Palavras-chave: Psicólogo, Formação, Educação.
\end{abstract}

\section{SCHOOL OF PSYCHOLOGY AT VENEZUELA: CURRICULAR STRUCTURE}

\section{Summary}

The paper is concerning to the academic formation of the psycho1ogist at Venezuela, studing the curriculum structure, taking in consideration the recommendations, suggestions and reflections presented in events that had influenced the teaching of Psychology and the action of the professional.

Key Words: Psychologist, Formation, Education.

La formación académica del profesional de la psicología ha constituido motivo de reflexión y discusión tanto en las universidades que administran la carrera como en distintos eventos: Congresos, foros, simposios, conferencias, seminarios, otros. Todos, con el propósito de encontrar la forma idónea, para proporcionar al futuro profesional un bagaje de conocimientos y experiencias que aporten las competencias necesarias para el ejercicio de sus funciones, ajustadas a las circunstancias individuales y sociales del hombre concreto. Se trata de ejercer la psicología como "Ciencia que se materializa en una profesión claramente vo1cada bacia la intervención con el decidido propósito de ser un instrumento que ayude al bienestar tanto de las personas concretas, grupos, instituciones y hasta sociedades." Blanco, Di Domenico, Pineda (1993).

En este orden de ideas, las Escuelas de Psicología, en Venezuela, se han preocupado por la actualización y adaptación de los planes de estudios en función a los cambios y exigencias que demanda la sociedad y evolución de la propia disciplina.

Con el propósito de enmarcar las reformas del pensum de estudio de Psicología, en el contexto venezolano, estimamos convenientemente hacer referencia a las orientaciones, recomendaciones, sugerencias y consideraciones, emanadas de algunos eventos que han influenciado el delineamiento de la enseftanza de la psicología.

Por razones de brevedad el siguiente cuadro recoge una síntesis de lo planteado en distintos momentos de la historia de la psicología como profesión.

\footnotetext{
${ }^{1}$ Este trabajo fue presentado en la mesa redonda: "Ensino de Psicologia: 'Reflexoes sobre la formação actual do Psicólogo"' V CONPE, Itajaí, 2000.

${ }^{2}$ Calle Libertad, Res. Tamarindo apt 12 D Piso 12 Torre A - 2101 Maracay, E. Aracua, Venezuela, castilho@ptvsa.com.
} 
FORMACIÓN DEL PSICÓLOGO: TEMA DE DISCUSIÓN

\begin{tabular}{|c|c|c|c|}
\hline Añ̄o & Instituciones/eventos & Propósito & Recomendaciones/Conclusiones \\
\hline 1945 & $\begin{array}{l}\text { Universidad de Harvard } \\
\text { Nombre unat comisión de } 12 \\
\text { notabies Pricóloges (Hilgard, } \\
\text { Thurstone, Thurdike, } \\
\text { Dollard, Camichael, entre } \\
\text { otros) }\end{array}$ & $\begin{array}{l}\text { Dar respuesta a cacstiones como: } \\
\text { - Definicióa del ámbito de la } \\
\text { Psicologia. } \\
\text { - Tipo de formación que dehe } \\
\text { impurtine y facultades respeasables } \\
\text { de administrarlas } \\
\text { Coordinación entre el trabajo del } \\
\text { Psiélogo y Psiquintra } \\
\text { - Otres }\end{array}$ & $\begin{array}{l}\text { Prevalece la orientación cienticifista en la } \\
\text { eatructura carricular. } \\
\text { Se incluyen lus disciplinas: Estadistica, } \\
\text { Maremática, Cálculo Fisico, Fisiologia, } \\
\text { Biologia y Psiquiatria. } \\
\text { Se perfila la función clínica del Psicólogo. }\end{array}$ \\
\hline 1949 & $\begin{array}{l}\text { Asociación de vetcranos de la } \\
\text { II guerra mandial y el } \\
\text { vervicio de Salud Póblica. } \\
\text { LSA. }\end{array}$ & $\begin{array}{l}\text { Requiere de las universidades el } \\
\text { disefio de un modelo de interveación } \\
\text { psicotógica para atender las } \\
\text { mecesidades de los sobrevivientes de } \\
\text { guerra }\end{array}$ & $\begin{array}{l}\text { Favorece la intervencióa clílea } \mathrm{c} \text { indisidual } \\
\text { como funciín prioritaria del psićlogo. }\end{array}$ \\
\hline 1958 & $\begin{array}{l}\text { Conferencia: Formación del } \\
\text { Psicílogo. } \\
\text { Miaml Beach } \\
\end{array}$ & $\begin{array}{l}\text { Disertar sobre nuevas peropectivas en } \\
\text { la formación del psicólogo }\end{array}$ & $\begin{array}{l}\text { - Se plantea la necesidad de ampliar el } \\
\text { campo de actuación profesional del } \\
\text { psicólogo. }\end{array}$ \\
\hline 1973 & $\begin{array}{l}\text { Conferencia de Yail } \\
\text { (Colerado) }\end{array}$ & $\begin{array}{l}\text { Dar respecsta a los cuestineuriosen } \\
\text { relación a la formación del psicólo- } \\
\text { go ca los diferentes aivelcs: } \\
\text { Pregrado y Postgrado. } \\
\text { - Otros }\end{array}$ & 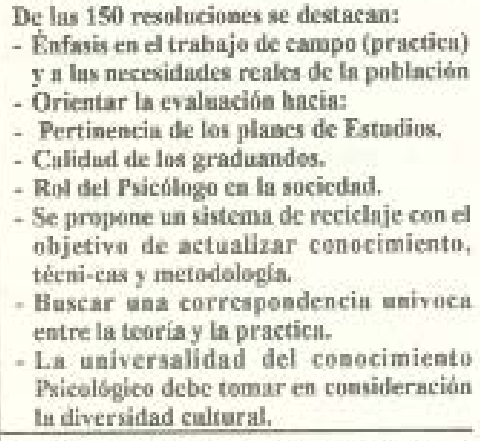 \\
\hline 1987 & $\begin{array}{l}\text { Cunlerencia de la APA } \\
\text { (Eniversidad de UTA) }\end{array}$ & 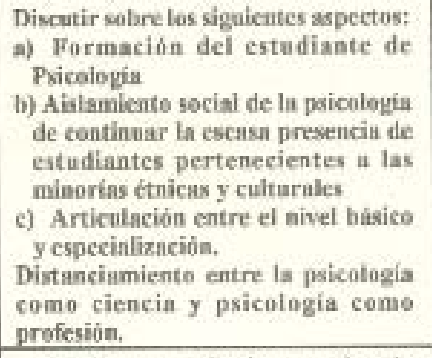 & 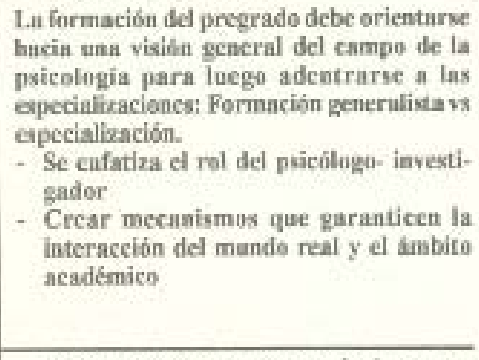 \\
\hline 1993 & $\begin{array}{l}\text { Simpesie: La formación del } \\
\text { paicáloge para el año } 2000 . \\
\text { XXIV congreso } \\
\text { interamericano de Psicologís. } \\
\text { Chile }\end{array}$ & $\begin{array}{l}\text { - Analizar y reflexionar sobre la } \\
\text { evolución de la psicologia y su } \\
\text { proyección ante las nuevas } \\
\text { exigencias sociales. } \\
\text { - Lu enseñanza de la paicología con las } \\
\text { universidlades }\end{array}$ & 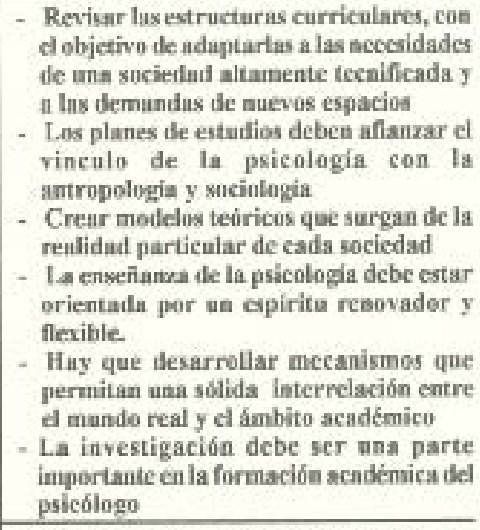 \\
\hline $\begin{array}{l}1997 \\
\text { a } 1999\end{array}$ & $\begin{array}{l}\text { XXVI Congreso } \\
\text { Interamericano de } \\
\text { Pricologia Brasil. } \\
\text { IV Coogreso Nacional de } \\
\text { Psicologia Escolar Brasil } \\
\text { XXVII Congreso } \\
\text { Internmericuno te Pxicologin } \\
\text { Caracas }\end{array}$ & & 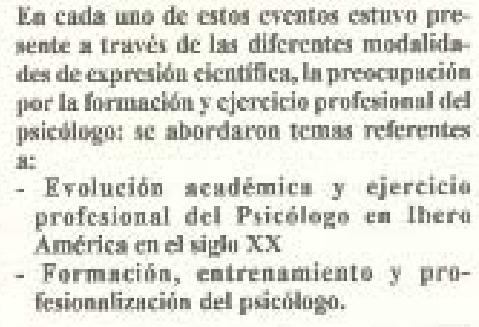 \\
\hline
\end{tabular}


Los planteamientos reseñados reflejan recurrente preocupación por:

- Labor y proyección del Psicólogo

- Formación Psicólogo - Investigador

- Universalidad del conocimiento psicológico vs diversidad cultural

- Problemática deI distanciamiento entre la psicologia como ciencia y psicologia como profesión.

- Orientación curricular: Cientificista vs Humanista

Intervención clínica individual vs grupal, colectiva Formación Generalista vs Especialista

Estos temas constituyen hoy motivo de discusión y quizás lo sean por mucho tiempo, dada la complejidad implícita en la formación profesional que se aspira, sea interdisciplinaria y multidisciplinaria en lo académico, investigación y compromiso social. Vezzete (1998) Dembo (1993) Yukimitsu (1999) Witter (1999). Cabe destacar la corriente de consenso en cuanto la necesidad de egresar psicólogos con una sólida formación como investigador.

Teniendo como referencia esta condensada sintesis nos proponemos presentar una sinopsis sobre la historia de la enseflanza de la psicologia en Venezuela.

\section{Reseña Histórica}

Las primeras Escuelas de Psicologia fueron creadas en los años 1957 por la Universidad Central de Venezuela (U.C.V) y en 1960 por la Universidad Católica Andrés Bello (UCAB).

En las cuatro décadas transcurridas, se ban generado procesos de renovación académica y administrativas. En esta ocasión es bará referencia a los procesos más relevantes llevados a cabo en las citadas universidades.

La carrera de Psicologia se inicia en la U. C. V. con el pensum de estudios que se resefla a continuación: 
PENSUM $1957(*)$

\begin{tabular}{|c|c|}
\hline$\underline{I}^{\text {ax }} \underline{\text { año }}$ & $\underline{2}^{\underline{\underline{\omega}}} \underline{\underline{A n} \theta}$ \\
\hline Pșicología General & Psicologia Evolutiva \\
\hline Lecturas de Psicología General & Prácticas de Psicología Evolutiva \\
\hline Lógica y Metodología & Psicología de la Personalidad \\
\hline Biología & Estadística \\
\hline Neurofisiologia & Inglés \\
\hline Prácticas de Neurofisiología & Pedagogia $(* *)$ \\
\hline Matemática & Prácticas de Psicología General \\
\hline \multicolumn{2}{|l|}{ Antropología y Sociologia $\left({ }^{* *}\right)$} \\
\hline$\underline{3} \underline{\Delta \tilde{n} o}$ & $\underline{4}^{+} A \tilde{n} 0$ \\
\hline \multicolumn{2}{|l|}{ Teoría y Práctica de Tests } \\
\hline Psicologia Diferencial & Psicología Clínica \\
\hline Psicología Social & Orientación Profesional \\
\hline Psicopatologia & Psicología Industrial \\
\hline Inglés & Seminario \\
\hline Didáctica $(* *)$ & Teorias Psicológicas \\
\hline Tests Proyectivos & Prácticas Docentes \\
\hline
\end{tabular}

$\left(^{*}\right)$ El Pensum se administró mediante dos modalidades: Plan A. Diurno, 4 años. Plan B. Nocturno. 5 años. Se egresa como Psicólogo Generalista

(**) Disciplinas del área de Ciencias Sociales, no especificas de la carrera.

En 1970 se reestructura el plan de estudios, etableciéndose un ciclo básico común y un ciclo aplicado. (Plan de estudios vigente).

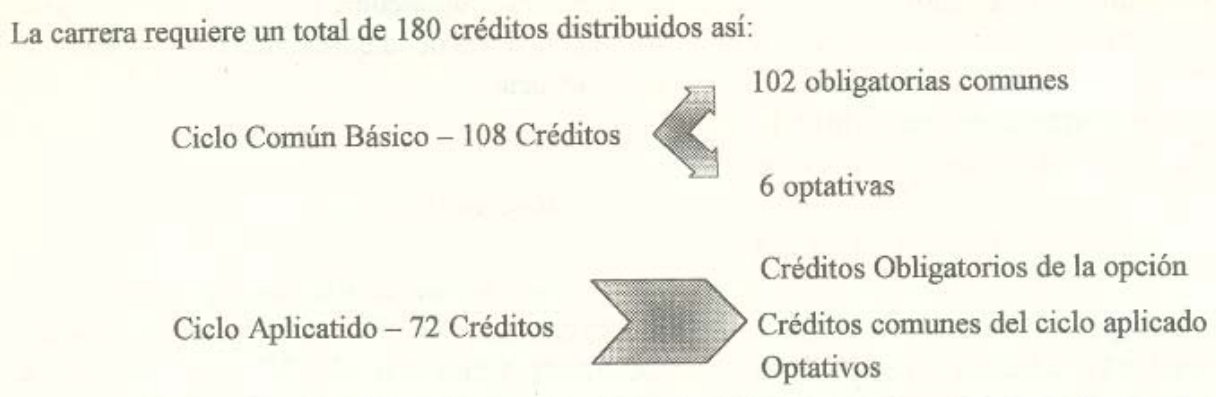

La propuesta curricular vigente está dirigida a formar psicólogo en correspondencia al siguiente perfil general:

a) Sensibilidad respecto a los problemas sociales, científicos que el desarrollo integral y progresista del pais, plantea a todos los sectores sociales, entre ellos los profesionales universitarios.

b) Actitud científica 
c) Capacidad para planificar científicamente su actuación

d) Capacidad para comprender el valor de la investigación y apreciar sus resultados para el perfeccionamiento de su desempeño profesional.

e) Capacidad para trabajar en equipo

f) Disposición para contribuir al progreso de la ciencia de la conducta.

Asignaturas Obligatorias Comnnes del Ciclo Básico

\begin{tabular}{|c|c|c|c|}
\hline SEMESTRE & ASIGNATURA & SEMESTRE & ASIGNATURA \\
\hline $1^{\circ}$ & $\begin{array}{l}\text { Psicología } \\
\text { General I } \\
\text { Estadística I } \\
\text { Teoría Social }\end{array}$ & $4^{\circ}$ & $\begin{array}{l}\text { PsicoIogía } \\
\text { Evolutiva } \\
\text { Psicometria I } \\
\text { Estructura Social } \\
\text { Venezolana }\end{array}$ \\
\hline $2^{\circ}$ & $\begin{array}{l}\text { Psicología } \\
\text { General II } \\
\text { Estadística II } \\
\text { Neurofisiología }\end{array}$ & $5^{\circ}$ & $\begin{array}{l}\text { Psicopatología } \\
\text { Psicometria II } \\
\text { Psicología Social }\end{array}$ \\
\hline $3^{\circ}$ & $\begin{array}{l}\text { Psicología } \\
\text { General III } \\
\text { Estadística III } \\
\text { Psicofisiología }\end{array}$ & $6^{\circ}$ & $\begin{array}{l}\text { Psicología } \\
\text { Experimental } \\
\text { Personalidad }\end{array}$ \\
\hline
\end{tabular}

\section{OPCIÓN: PSICOLOGÍA ESCOLAR}

Estudio Psicológico I

Ps. Aplicada a la Educación I

Ps. Aplicada a la Educación II

Ps Aplicada a la Educación III

Estudio Psicológico II

Sist. Educativo Venezolano II

Tecn. De interv. En Ps. Escolar I

Tecn. De interv. En Ps. Escolar III

Pasantía

Tecn. De interv. En Ps. Escolar II

Sist. Educativo Venezolano II

Seminario de Investigación I

Seminario de Investigación II

OPCIÓN: PSICOLOGÍA CLÍNICA

Introducción a la metodologia del Análisis Experimental de la Conducta Introducción a la Psicologia Clínica

Psicologia Clínica Infantil

Psicologia Clínica de Adultos

Neuropsiquiatria

Teoria de Tratamientos Psicológicos

Prácticas Clínica I

Prácticas Clínicas II

Retardo en el Desarrollo

Rehabilitación

Terapia Conductual

Criminologia

Prácticas Clínicas III

Pasantías Institucionales

Prácticas Clínicas IV

Seminario de Investigación
OPCIÓN: PSICOLOGÍA INDUSTRIAL

Instrumentos Psicométricos

Teoría Organizacional

Psicologia Organizacional

Organización, Diseño y medida del trabajo

Selección de Personal

Economía Venezolana

Entrenamiento de Personal

Relaciones Laborales

Sistema de Personal

Pasantía

Técnicas de Evaluación y Remuneración de

Personal

Metodologia de Investigación en Psicologia

Industrial

Desarrollo Organizacional

Seminario de Investigación

OPCIÓN: ASESORAMIENTO

PSICOLÓGICO Y ORIENTACIÓN

Instrumentos Psicométricos

Principios de Orientación

Psicologia Vocacional

Técnicas de Entrevista

Técnicas de Información Ocupacional

Técnicas de Orientación de Grupo

Seminario de Investigación en orientación

Estudio de Casos

Pasantía I

Organización de Servicios

Pasantía II

Seminario de Investigación 


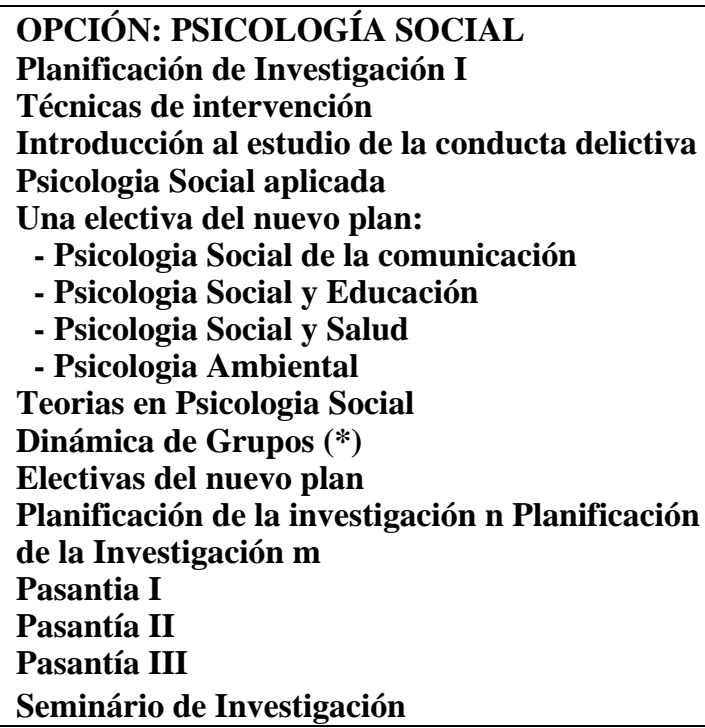

$\mathrm{Al}$ analizar la oferta académica en su conjunto cabe destacar lo siguiente:

- Esta subyacente una concepción cientificistatecnológica dirigida a la formación de un especialista de la conducta humana.

- Poco énfasis en la formación del Psicólogo investigador

- No se incluyen disciplinas relacionadas con las áreas emergentes en el campo de la Psicologia (penal, Deporte, Forense, Comunitario)

- La oferta de materias están centradas en las especialidades respectivas, observándose poca tendencia hacia la multidisciplinaridad e interdisciplinaridad.

En relación a la Universidad Católica Andrés Bello, los Estudios de Psicologia han seguido la siguiente trayectoria.

- Se crea la Escuela de Psicologia

- Formación de Psicólogo Generalista

- Régimen Anual

- Lacarrera tiene una duración de 4 anos

- El Pensum de estudios se administra según el siguiente esquema:

$\cdot 1^{\mathrm{er}}, 2^{\mathrm{do}}$ y $3^{\mathrm{er}}$ afio: Materias de Carácter Generales

- $4^{\text {to }}$ y $5^{\text {to }}$ año disciplinas especificas de las áreas: Clínica, Escolar, Orientación e Industrial.

- No se incluyen Disciplinas Optativas

\section{9-2001}

- Está en proceso la reestructuración del pensum de estudio con el propósito de:

- Orientar el curriculo hacia uma formación dirigida a la intervención grupal, colectiva:

Psicólogo Clínico x de la salud

Psicólogo Industrial x Laboral

Psicólogo Escolar x Psicólogo del Desarrollo 
Organizacional

- Atender las áreas emergentes dentro del ámbito de la Psicologia: Deporte, Jurídica, Forense, Comunitaria, otra.

- Flexibilizar el pensum de estudio incorporando disciplinas optativas

- Se mantiene la formación de Psicólogo generalista.

En el planteamiento curricular de ambas escuelas de Psicologia se puede apreciar enfoques similares en cuanto a:

- Orientación Cientificista - Tecnológica

- Énfasis en el enfoque clínico

- La matriz curricular no apuntala hacia la interdisciplinaridad

- A manera de conclusión podemos señalar que las estructuras curriculares que rigen los estudios de Psicologia en ambas Universidades (Universidad Católica Andrés Bello y Universidad Central de Venezuela) se orientan desde un enfoque academicista, tendencia predominante en la mayoria de las escuelas de Psicologia Latinoamericanas. Dembo (1993)

- De igual manera, cabe destacar, que las recomendaciones emanadas desde distintos escenarios, referente a la necesidad de profundizar la formación del PsicólogoInvestigador, abrir el Pensum de estudio hacia la interdisciplinaridad, a la actuación grupal, comunitaria y un mayor acercamiento entre la academia y la realidad concreta, no ha tenido la resonancia esperada, en las propuestas curriculares aqui descritas.

\section{MENCIÓN: PSICOLOGIA ESCOLAR PERFIL DEL PSICÓLOGO ESCOLAR}

A través de su formación el Psicólogo Escolar habrá desarrollar destrezas que lo capaciten para actuar en cinco roles principales:

1) Como Investigador: Estudiar exhautivamente la realidad social y educativa que permitan el conocimiento y comprensión de la complejidad del hecho educativo;

2) Como Evaluador: El conocimiento de la magnitud de un problema especifico es la base que permite emprender acciones eficaces;

3) Como Planificador: Deberá poner en práctica todo sus conocimientos técnicos acerca de la ciência Psicológica y aspectos sociales de su cultura;

4) Como Asesor: Contribuir a producir los cambios necesarios a través de equipos multidisciplinarios;

5) Como Interventor: Atención de problemáticas especificas tanto individuales como grupales. 


\begin{tabular}{|l|}
\multicolumn{1}{|c|}{ Matriz Curricular } \\
\multicolumn{1}{|c|}{ Opción: Psicologia Escolar } \\
Estudio Psicológico I \\
Ps. Aplicada a la Educación I \\
Ps. Aplicada a la Educación II \\
Ps Aplicada a Ia Educación III \\
Estudio Psicológico II \\
Sist. Edqcativo Venezolano II \\
Tecn. De interv. En Ps. Escolar I \\
Tecn. De interv. En Ps. Escolar III \\
Pasantía \\
Tecn. De interv. En Ps. Escolar II \\
Sist. Educativo Venezolano II \\
Seminario de Investigación I \\
Seminario de Investigación II \\
\hline
\end{tabular}

\section{Consideraciones de la Oferta Académica}

Del análisis de la oferta académica se puede concluir lo siguiente:

- La formación académica está dirigida a capacitar al estudiante desde una óptica centrada en las variables internas del niño, adolescente y joven:

- Desarrollo cognitivo

- Madurez emocional

- Autoestima

- Motivación

- Diferencias individuales

- Evaluación Psicopedagógica

- Estilo de aprendizaje

- Otros

- No se enfatiza las variables externas que afectan, por igual, el proceso de escolaridad, en el más amplio sentido:

- Concepción Antropológica

- Concepción Pedagógica de los planes de estúdio

- Procedimientos metodológicos y didácticos

- Interrelaciones Personales

- Contexto Socio-Histórico

- El rol del investigador no se ejercita, lo suficiente, durante el periodo de formación.

- El Plan de Estudio no incluye disciplinas como Antropologia Cultural, Planificación Curricular, Sociologia, Formación Docente, Administración Escolar y Otras que contribuyan a una visión global multidisciplinaria del área de actuación del Psicólogo Escolar. 


\section{REFERENCIAS}

BLANCO. A, DIDOMENICO. C Y PINEDA. Psicología: Ciencia y Compromiso. XXIV Congreso Interamericano de Psicología. Chile: 1998.

DEMBO.M. Estrategias y Contenidos en la formación del Psicólogo. XXIV Congreso Interamericano de Psicología. 1993.

FORO. Historical Perspective an current Devepeloment of Psycology in Argentina, Venezuela and Brazil. Revista Interamericana de Psicología. 1998, Vol 32. ํำ 1.

WPEZ,M. El Psicólogo Escolar y la Escuela Básica. Escuela de Psicología. Venezuela: Universidad Central de Venezuela, 1983.

Universidad Católica Andrés Beno. Pensum de Estudio. Venezuela: Escuela de Psicología, 1960.

Universidad Central de Venezuela. Pensum de Estudio. Venezuela: Escuela de Psicología, 1970.

VEZZETTI.H. La Psicología del fin de Siglo a la Luz de su historia. Revista Interamericana de Psicología, 998. Vo1 51 (1).

WESCHLER,M.S. Psicología Escolar: Pesquisa, Formação e Práctica. Alínea, 1996.

WITTER,C. Encino de Psicología. Alínea, 1999.

VICENTELLI.H. Proyección del Psicólogo Escolar en la Comunidad Educativa de Maracay. Venezuela. Psicología Escolar e Educacional. 1996. Vol 1. №1. XXVII Congreso Interamericano de Psicología. Caracas - Venezuela: 1999.

\section{ENDEREÇO}

Hermínia Vicentelli

Rua Calle Libertad, 2101

Res. Tamarindo, apto. 12D - Piso 12 - Torre A

Maracay - Venezuela 